\title{
Treatment results for hypopharyngeal cancer by different treatment strategies and its secondary primary- an experience in Taiwan
}

\author{
Morgan Fu-Ti Chang ${ }^{1}$, Hung-Ming Wang ${ }^{2,5,6}$, Chung-Jan Kang ${ }^{3,5}$, Shiang-Fu Huang, 3, , Chien-Yu Lin ${ }^{4,5,7}$, \\ Kang-Hsing Fang ${ }^{4,5,7}$, Eric Yen-Chao Chen ${ }^{4,5}$, I-How Chen ${ }^{3,5}$, Chun-Ta Liao ${ }^{3,5}$, Joseph Tung-Chieh Chang ${ }^{4,5,6^{*}}$
}

\begin{abstract}
Purpose: The aim of this study was to evaluate treatment results in our hypopharyngeal cancer patients.

Patients and Methods: A total of three hundred and ninety five hypopharyngeal cancer patients received radical treatment at our hospital; $96 \%$ were male. The majority were habitual smokers (88\%), alcohol drinkers (73\%) and/or betel quid chewers (51\%). All patients received a CT scan or MRI for tumor staging before treatment. The stage distribution was stage I: 2 (0.5\%); stage II: 22 (5.6\%); stage III: 57 (14.4\%) and stage IV: 314 (79.5\%). Radical surgery was used first in 81 patients (20.5\%), and the remaining patients (79.5\%) received organ preservation-intended treatment (OPIT). In the OPIT group, 46 patients received radiotherapy alone, 156 patients received chemotherapy followed by radiotherapy (CT/RT) and 112 patients received concomitant chemo-radiotherapy (CCRT).

Results: The five-year overall survival rates for stages I/II, III and IV were $49.5 \%, 47.4 \%$ and $18.6 \%$, respectively. There was no significant difference in overall and disease-specific survival rates between patients who received radical surgery first and those who received OPIT. In the OPIT group, CCRT tended to preserve the larynx better ( $p=$ 0.088), with three-year larynx preservation rates of $44.8 \%$ for CCRT and $27.2 \%$ for CT/RT. Thirty-seven patients developed a second malignancy, with an annual incidence of $4.6 \%$.

Conclusions: There was no survival difference between OPIT and radical surgery in hypopharyngeal cancer patients at our hospital. CCRT may offer better laryngeal preservation than RT alone or CT/RT. However, prospective studies are still needed to confirm this finding. Additionally, second primary cancers are another important issue for hypopharyngeal cancer management.
\end{abstract}

\section{Introduction}

Patients with carcinoma of the hypopharynx frequently have advanced disease at the time of presentation. These patients have some of the worst prognoses of all head and neck cancer patients, and combined-modality therapy is usually required to achieve a cure. The conventional treatment for advanced, but resectable, cases has been surgery followed by post-operative adjuvant therapy, and five-year survival rates vary from $10 \%$ to $60 \%$ [1-5]. Recently, the integration of chemotherapy and radiotherapy was investigated for organ preservation

\footnotetext{
* Correspondence: cgmhnog@gmail.com

${ }^{4}$ Department of Radiation Oncology, Chang Gung Memorial Hospital at Linkou, Taoyuan, Taiwan

Full list of author information is available at the end of the article
}

in patients with locally advanced hypopharyngeal cancers. The results of these prospective trials were encouraging; they indicated that the larynx could be preserved using combined chemotherapy and radiotherapy without compromising overall survival rates [6-10].

Two phase III trials [11,12] of sequential chemotherapy and radiotherapy for resectable laryngeal or hypopharyngeal cancer revealed survival rates similar to those achieved with surgery and post-operative irradiation, but the larynx was preserved for many patients in the former group. On the other hand, a meta-analysis [13] of six trials comparing induction chemotherapy and radiotherapy with alternating or concomitant chemoradiotherapy (CCRT) revealed a hazard ratio of 0.91 (0.79-1.06) in favor of the latter. This analysis also 
showed a five-year survival benefit of $32 \%$ - $40 \%$ when chemotherapy was added concomitantly to radiotherapy. Growing evidence suggests that CCRT may improve loco-regional tumor control in locally advanced head and neck cancers and, more importantly, improve survival rates compared with the sequential regimen or radiotherapy alone $[14,15]$.

To the best of our knowledge, no existing data demonstrate whether CCRT could enhance organ preservation in hypopharyngeal cancer patients. In this article, we present treatment results for our hypopharyngeal cancer patients. Furthermore, we determine whether concomitant use of chemotherapy offers the best chance of organ preservation.

\section{Patients and Methods}

From January 1994 to May 2004, 430 hypopharyngeal cancer patients were referred for radiotherapy evaluation. We excluded 35 patients who refused radical therapy, leaving 395 patients for analysis. All patients received computed tomography scans or magnetic resonance imaging (MRI) for staging prior to radical treatment. Initially, 81 patients $(20.5 \%)$ first received radical surgery, and the remaining patients (79.5\%) underwent organ preservation-intended therapy (OPIT). Treatment decisions were based on the preference of the serving physician and/or patient. In the group that initially received radical surgery, patients with risk factors such as positive pathological margin, more than two lymph node metastases or extracapsular extension of the lymph nodes also received concomitant chemotherapy when post-operative radiotherapy was performed. In the OPIT group, 47 patients received radiotherapy (RT) alone, 188 patients received induction chemotherapy followed by radiotherapy $(\mathrm{CT} / \mathrm{RT})$ and 79 patients received CCRT.

The chemotherapy (CT) regimen, PTL, was detailed in our previous report [16]. In brief, it consists of $50 \mathrm{mg} /$ $\mathrm{m}^{2}$ cisplatin (P) on Day 1 , followed by $800 \mathrm{mg} / \mathrm{m}^{2}$ oral tegafur $(\mathrm{T})$ per day and $60 \mathrm{mg}$ oral leucovorin $(\mathrm{L})$ per day for 14 days. The CT was administered at outpatient clinics in 14-day cycles. In the CT/RT group, re-evaluation after three cycles of chemotherapy led to the termination of CT if tumor responses were less than partial responses. Otherwise, PTL regimens were continued for up to six cycles before radiotherapy. Patients achieving at least good partial responses at the primary site after neoadjuvant chemotherapy received radiotherapy or chemo-radiotherapy for organ preservation.

Radiotherapy was performed by three-field technique; it consisted of conventional bilateral opposing fields with a matching anterior lower neck portal. The daily fractionation size was 1.8 or $2 \mathrm{~Gy}$, with five fractions per week. The median dose to the gross tumor volume was 68.4 Gy (range: 60-76 Gy), and to clinical target volume was 45 Gy (range 45-46 Gy). The planning target volume was created by adding 5-7 mm margin from clinical target volume. For the group receiving radical surgery first, the post-operative radiotherapy dose was 60-68.4 Gy, depending on the pathology risk factor; for the OPIT group, the dose range was 68.4-76 Gy. The spinal cord was shielded by customerized cerrobend block or multi-leaf collimator after 45-46 Gy and the posterior neck regions were boosted with a $9-12-\mathrm{MeV}$ electron beam for an additional 14-24 Gy in 7-12 fractions, according to the status of the regional lymph nodes.

In the organ preservation group, planned neck dissection was not routinely performed. Salvage surgery or neck dissection was undertaken when any residual lesion was noted in the post-treatment evaluation, which was usually performed three months after radical treatment or in the case of tumor progression.

All patients were followed in the clinic every one to two months for the first two years, and then every three to four months in the third to fifth years. Computer tomography scans, bone scans, chest X-rays, SMA and $\mathrm{CBC}$ were scheduled routinely (at least annually) for at least the first three years post-treatment to detect recurrence. The primary endpoint of our study was overall survival rate, with a second endpoint of disease-specific survival rate (DSS). The duration of survival was defined as the time from the first date of radical treatment to the date of the event, which was death for the overall survival rate or tumor-related mortality for DSS. For survival with a preserved larynx (OSP), the event was defined as death or total laryngopharyngectomy. Locoregional or distant control meant that no recurrence could be verified by pathological examination or progressive changes in serial image studies when no tissue proof was available. Statistical Package for the Social Sciences software (SPSS Inc., Chicago, IL) was used for statistical analysis. The Kaplan-Meier method was used to estimate survival rates with the log-rank test for subgroup analyses. A p-value of $<0.05$ was considered significant. Multivariate analyses were assessed using the Cox-regression model.

\section{Results}

\section{Patient population}

The characteristics of all patients are listed in Table 1. Ninety-six percent were male, and the median age was 56 years (range: $15-87)$. The majority of patients were habitual smokers (86.6\%), alcohol drinkers (69.6\%) and/ or betel quid chewers (47.1\%). All patients were restaged according to the AJCC 2002 staging system. The stage distribution was as follows: stage I: $2(0.5 \%)$, stage II: 22 (5.6\%), stage III: $57(14.4 \%)$ and stage IV: 314 (79.5\%). 
Table 1 Patient characteristics

\begin{tabular}{|c|c|c|c|c|}
\hline & $\begin{array}{l}\text { Case Numbers } \\
\text { (percentage) }\end{array}$ & $\begin{array}{l}\text { Radical } \\
\text { surgery } \\
\text { group }\end{array}$ & $\begin{array}{l}\text { Organ } \\
\text { preservation } \\
\text { group }\end{array}$ & P-value \\
\hline Age, years & & & & 0.035 \\
\hline$\leqq 55$ & $188(47.6 \%)$ & 47 & 141 & \\
\hline$>55$ & 207 (52.4\%) & 34 & 173 & \\
\hline Gender & & & & 0.176 \\
\hline Male & $380(96.2 \%)$ & 80 & 300 & \\
\hline Female & 15 (3.8\%) & 1 & 14 & \\
\hline Smoking & & & & 0.856 \\
\hline Yes & $342(86.6 \%)$ & 71 & 271 & \\
\hline No & $53(13.4 \%)$ & 10 & 43 & \\
\hline $\begin{array}{l}\text { Alcohol } \\
\text { drinking }\end{array}$ & & & & 0.869 \\
\hline Yes & 275 (69.6\%) & 57 & 218 & \\
\hline No & $120(30.4 \%)$ & 24 & 96 & \\
\hline \multicolumn{5}{|l|}{$\begin{array}{l}\text { Betel nut } \\
\text { chewing }\end{array}$} \\
\hline Yes & $186(47.1 \%)$ & 41 & 145 & 0.533 \\
\hline No & 209 (52.9\%) & 40 & 169 & \\
\hline T stage & & & & 0.012 \\
\hline $\mathrm{T} 1$ & 19 (4.8\%) & 4 & 15 & \\
\hline $\mathrm{T} 2$ & 71 (18\%) & 11 & 60 & \\
\hline T3 & 73 (18.5\%) & 6 & 63 & \\
\hline T4 & $232(58.7 \%)$ & 60 & 172 & \\
\hline N stage & & & & 0.300 \\
\hline NO & $113(28.6 \%)$ & 20 & 93 & \\
\hline N1 & 73 (18.5\%) & 12 & 61 & \\
\hline N2 & 154 (39\%) & 39 & 115 & \\
\hline N3 & 55 (13.9\%) & 10 & 45 & \\
\hline $\begin{array}{l}\text { Overall } \\
\text { Stage }\end{array}$ & & & & 0.013 \\
\hline I & $2(0.5 \%)$ & 0 & 2 & \\
\hline$\|$ & 22 (5.6\%) & 2 & 20 & \\
\hline III & 57 (14.4\%) & 4 & 53 & \\
\hline IV & $314(79.5 \%)$ & 75 & 239 & \\
\hline
\end{tabular}

\section{Overall survival and disease-specific survival}

The median follow-up time was 5.09 years. At the time of analysis, 269 patients had died: of these, 185 died of local disease, 35 died of distant metastasis and 49 died of a second primary tumor or other intercurrent disease. The five-year overall survival rate for all patients was $24.8 \%$. The five-year overall survival rates for stages I/II, III and IV were $49.5 \%, 47.4 \%$ and $18.6 \%$, respectively (p $<0.001$ ). The five-year DSS rates for stages I/II, III and IV were $67.4 \%, 53.5 \%$ and $25.5 \%$, respectively ( $\mathrm{p}<$ 0.001 ). The results of subgroup analyses are illustrated in Table 2.

There was no significant difference in the overall survival rate or DSS rate between the group of patients receiving radical surgery first and the organ-preservation intended treatment group. The five-year overall survival rate and DSS rate were $18.8 \%$ and $24.2 \%$ in the radical surgery-first group and $27 \%$ and $35.9 \%$ in the OPIT group, respectively (Figure $1 \& 2$ ). There was no significant difference in the survival rate based on the type of combination between chemotherapy and radiotherapy. The five-year overall survival rate and DSS rate were $20.5 \%$ and $29.2 \%$ for the CT/RT group and $43.1 \%$ and $53 \%$ for the CCRT group, respectively ( $\mathrm{p}=0.200$ for overall survival rate and $p=0.397$ for DSS). Besides, when confine the patients into stage III and IV, there is no significant difference between OPIT group and radical surgery group in overall survival rates and diseasefree survival rates $(\mathrm{p}$-value $=0.449$ and 0.427 respectively).

The five-year overall survival rate was $45.9 \%$ and the DSS rate was $54.4 \%$ in patients without evidence of recurrence. Recurrent patients who suffered from locoregional failures had better prognoses than those with distant failures (Table 2). T-stage, $\mathrm{N}$-stage and recurrence were all independent predictors of overall survival and DSS after multivariate analysis (Table 3).

For patients who only experienced loco-regional recurrences, salvage surgery with or without adjuvant radiotherapy and chemotherapy was given under certain conditions. The five-year DSS rate was $27.8 \%$, and the overall survival rate was $19.6 \%$. Chemotherapy was given to patients with distant metastasis with or without locoregional control and good performance status, and to patients with supportive care but with poor performance status. However, none of these patients survived longer than three years. The median survival time for patients with distant metastasis and without loco-regional control was 1.4 years; patients with recurrence at both distant and loco-regional sites survived for an average of 1.19 years.

\section{Organ preservation}

In the organ preservation group, 93 patients (29.6\%) survived with a preserved larynx at three years. There were no significant differences in patient characteristics between $\mathrm{C} / \mathrm{T}+\mathrm{RT}$ and CCRT except for less betel nut use in CCRT patients. Patients in early T-stage or Nstage had higher rates of larynx preservation. Smoking, alcohol drinking or betel quid chewing were not important factors for organ preservation. However, patients who received concomitant chemotherapy had a higher chance of survival with a preserved larynx when compared with patients who received induction chemotherapy (CT/RT; $37 \%$ vs. $18 \%$ of 4 -year OSP, p $=0.041$; Figure 3).

\section{Second primary malignancy}

During follow-up, 37 patients experienced a second primary malignancy. There were sixteen head and neck 
Table 2 Prognostic factors for survival rates, univariate analysis

\begin{tabular}{|c|c|c|c|c|c|}
\hline & Numbers (n) & 5-yr OS rate (\%) & p-value & 5-yr DSS rate (\%) & p-value \\
\hline Age, years-old & & & 0.747 & & 0.961 \\
\hline$\leqq 55$ & 188 & 25.5 & & 35.2 & \\
\hline$>55$ & 207 & 24.4 & & 31.4 & \\
\hline Smoking & & & 0.029 & & 0.075 \\
\hline Yes & 342 & 22.5 & & 30.3 & \\
\hline No & 53 & 41.7 & & 49.8 & \\
\hline Alcohol drinking & & & 0.081 & & 0.158 \\
\hline Yes & 275 & 22.6 & & 30.8 & \\
\hline No & 120 & 29.9 & & 37.3 & \\
\hline Betel nut chewing & & & 0.360 & & 0.159 \\
\hline Yes & 186 & 24.4 & & 32.2 & \\
\hline No & 209 & 25.0 & & 31.3 & \\
\hline T-stage & & & $<0.001$ & & $<0.001$ \\
\hline $\mathrm{T} 1$ & 19 & 54.3 & & 68.6 & \\
\hline $\mathrm{T} 2$ & 71 & 38.1 & & 45.1 & \\
\hline T3 & 69 & 30.7 & & 38.2 & \\
\hline T4 & 232 & 17.2 & & 24.4 & \\
\hline $\mathrm{N}$-stage & & & $<0.001$ & & $<0.001$ \\
\hline NO & 113 & 32.4 & & 40.6 & \\
\hline N1 & 73 & 36.4 & & 43.6 & \\
\hline N2 & 154 & 20.6 & & 30.1 & \\
\hline N3 & 55 & 0 & & 0 & \\
\hline Stage & & & $<0.001$ & & $<0.001$ \\
\hline$|/| \mid$ & 24 & 49.5 & & 67.4 & \\
\hline III & 57 & 47.4 & & 53.5 & \\
\hline IV & 314 & 18.6 & & 25.5 & \\
\hline Treatment & & & 0.229 & & 0.069 \\
\hline Radical surgery first & 81 & 18.8 & & 24.2 & \\
\hline Organ preservation & 314 & 27.0 & & 35.9 & \\
\hline
\end{tabular}

cancers (five tongue, four oropharynx, three mouth floor, two buccal region, one larynx and one submandibular gland), twelve esophageal cancers, twelve lung cancers, six bladder cancers and one colon cancer. The median time to the development of the second primary malignancy was 2.64 years, with a $4.6 \%$ rate of annual incidence (Figure 4).

\section{Discussion}

Symptoms of hypopharyngeal cancers occur late, so most of them are diagnosed at an advanced stage. Almost $80 \%$ of our patients presented with stage IV disease. Among head and neck cancers, hypopharyngeal cancer has the worst prognosis. The five-year overall survival rate was $24.8 \%$ in our series, which is comparable to results from other studies where overall survival rates varied from 10 to $60 \%$ [1-3,6-10,12,17-23].

The conventional treatment for locally advanced but resectable head and neck cancers has been surgery with post-operative adjuvant therapy depending on the risk factors for recurrence after surgery. Radiotherapy, however, is the treatment of choice for unresectable or medically inoperable patients. To improve survival rates and preserve organs, a combination of chemotherapy and radiotherapy was introduced. Most retrospective studies of head and neck cancers included various subsites (Table 4). Some series revealed a significant rate of organ preservation with similar survival rates between surgery and chemo-radiotherapy in head and neck cancer patients $[1,4,6-12,15,16,18,19,23-27]$, especially for laryngeal cancer. In this study, we separated the entire patient population into two main treatment groups: radical surgery or organ preservation. There was no significant difference in the overall survival rate and DSS rate between patients who received radical surgery first and patients in the organ preservation group. However, patients who survived longer than three years had a $33.2 \%$ larynx preservation rate in the latter group.

Two large phase III randomized trials demonstrated that induction chemotherapy followed by definite radiotherapy $(\mathrm{CT} / \mathrm{RT})$ yielded survival rates similar to those in patients receiving surgery and irradiation for laryngeal 


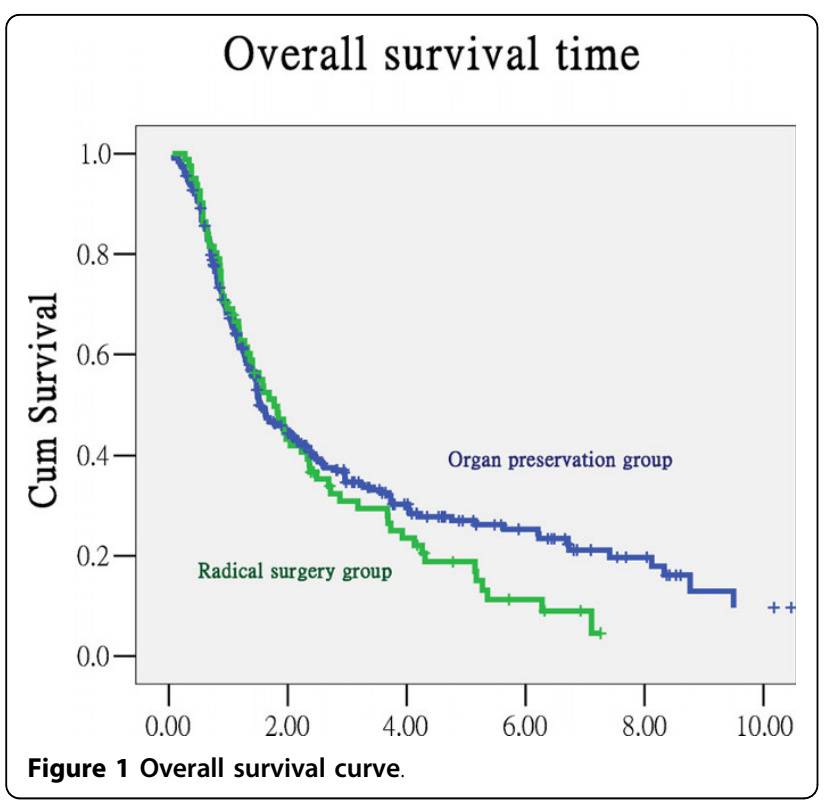

and pyriform sinus cancer, respectively $[11,12]$. The rationale for using induction chemotherapy is the identification of patients for radiotherapy according to the high predictability of subsequent radiotherapy response based on the response to chemotherapy. Therefore, induction chemotherapy could be used as a surrogate for patient selection to identify patients who are eligible for organ preservation. This procedure could avoid the inevitable severe complications for patients who receive high-dose RT followed by salvage surgery.

However, the results of a recent RTOG study of laryngeal cancer patients [11] challenged the role of induction chemotherapy in selecting the "right" patients for organ preservation. Concomitant chemo-radiotherapy can achieve better rates of organ preservation than induction chemotherapy selection followed by radiotherapy. Furthermore, in this study, eleven patients selected for radical surgery due to a poor response to induction chemotherapy did not accept radical surgery, so they received chemotherapy and radiotherapy. All of these patients achieved complete remission after radical treatment and, consequently, only one patient required a laryngectomy. Although the number is small and there may be some bias in the patients' treatment choices, the use of induction chemotherapy as a predictor of organ



preservation needs further study, especially in an era where more patients are choosing CCRT.

Concomitant chemotherapy may contribute to the radiosensitizing effect of radiotherapy and thus improve tumor control. A large meta-analysis showed that the survival rate increased significantly when chemotherapy was added to the treatment of head and neck cancers [13]. Although the heterogeneity of these 63 trials (including 10741 patients) limited the identification of conclusive results, chemotherapy given concomitantly with radiotherapy still had substantial benefits, corresponding to an absolute five-year survival benefit of $\mathbf{8 \%}$. Our study also found that patients who received CCRT had higher rates of survival with larynx preservation ( $44.8 \%$ at three years). Although there was no significant difference in overall survival, the use of CCRT allows the possibility of larynx preservation, which may have an impact on a patient's social activity and quality of life.

In retrospective trials of radiotherapy versus surgery, there is always the possibility of strong selection bias: usually the surgeons get the "better" patients because their patients need to be operable and/or resectable. In this study, a similar bias may have occurred. However, the OPIT group did not show a worse tumor control or survival rate than surgical group, and some large

Table 3 Multivariate analysis

\begin{tabular}{|c|c|c|c|c|c|c|}
\hline & \multicolumn{2}{|r|}{ T-stage } & \multicolumn{2}{|r|}{$\mathrm{N}$-stage } & \multicolumn{2}{|r|}{ Recurrence } \\
\hline & $p$-value & Hazard ratio $(95 \% \mathrm{Cl})$ & $p$-value & Hazard ratio $(95 \% \mathrm{Cl})$ & p-value & Hazard ratio $(95 \% \mathrm{Cl})$ \\
\hline$\overline{5 \text {-yr overall survival rate }}$ & $<0.001$ & $\begin{array}{l}0.332 \\
(0.169-0.652)\end{array}$ & $<0.001$ & $\begin{array}{l}0.321 \\
(0.218-0.470)\end{array}$ & 0.013 & $\begin{array}{l}0.503 \\
(0.32-0.790)\end{array}$ \\
\hline 5-yr disease-specific survival rate & 0.003 & $\begin{array}{l}0.325 \\
(0.151-0.699)\end{array}$ & $<0.001$ & $\begin{array}{l}0.290 \\
(0.189-0.445)\end{array}$ & 0.004 & $\begin{array}{l}0.435 \\
(0.264-0.717)\end{array}$ \\
\hline
\end{tabular}




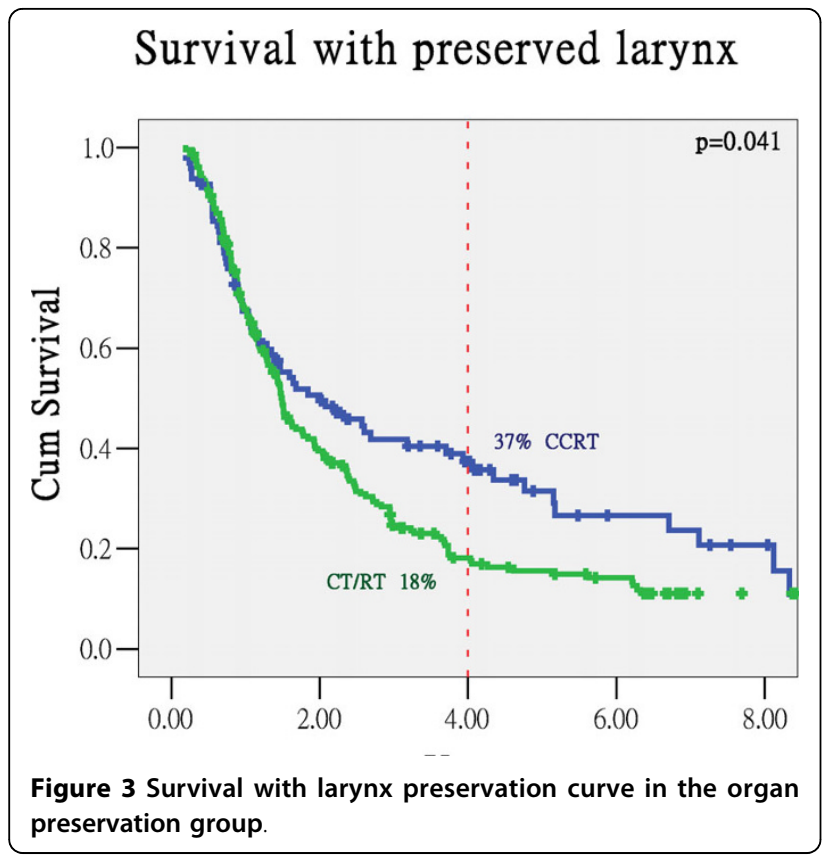

unresectable tumors were included in the OPIT group. Prospective studies would be valuable in addressing these issues.

Most patients in our study relapsed at loco-regional sites, and their five-year overall survival rate was only $19.6 \%$, which suggests that conventional radiotherapy techniques using bilateral opposing fields may compromise radiation dose coverage of the target after blocking of the spinal cord at doses of 46-50 Gy. Some studies of recent modern radiotherapy techniques such as intensity-modulated radiotherapy (IMRT) with concomitant chemotherapy yielded promising loco-regional control rates as well as disease-free and overall survival rates for hypopharyngeal cancer $[2,28,29]$. Some studies also

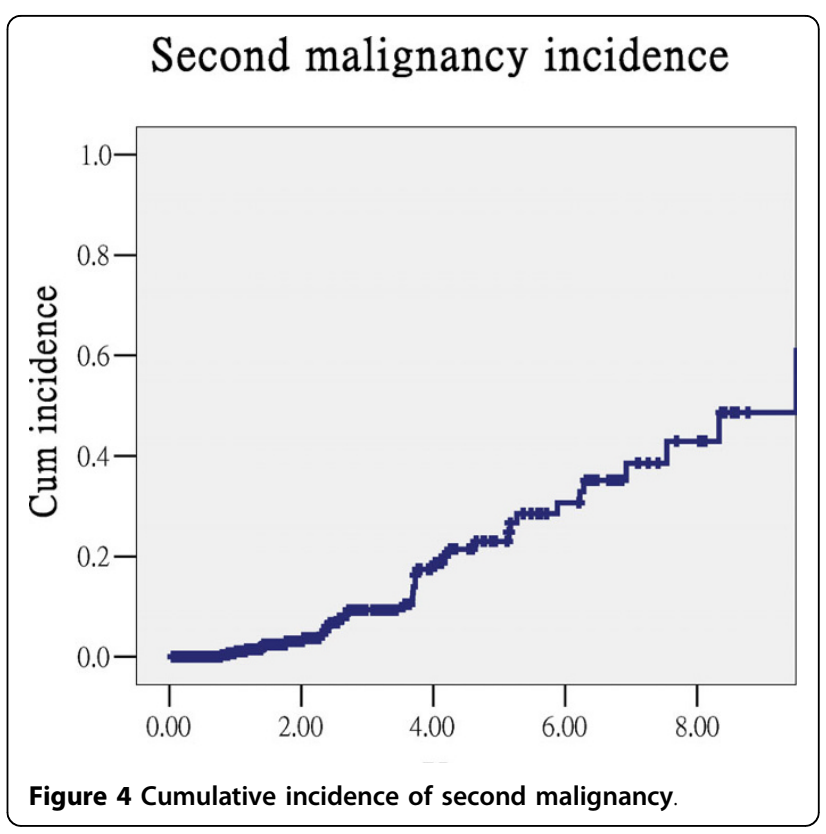

revealed that it is possible to decrease the severity of late toxicities such as dysphagia and aspiration using IMRT to spare the larynx and swallowing muscles $[30,31]$.

Second primary cancers were a major cause of death in this study, with an annual incidence rate of $4.6 \%$. The median time to the development of a second primary malignancy was 2.64 years. This incidence is similar to that reported in our previous study on oral cavity cancer [16], but the occurrence sites are slightly different. In oral cavity cancer, the most common second primary area of occurrence is the head and neck region, especially the oral cavity area (70.3\%). However, in this study, about $60 \%(21 / 37)$ of cancers occurred below the clavicle despite all of the patients having similar habits

Table 4 Organ preservation studies of head-and-neck cancers

\begin{tabular}{|c|c|c|c|c|c|c|}
\hline Author & $\begin{array}{l}\text { Year of } \\
\text { collection }\end{array}$ & $\begin{array}{l}\text { Case } \\
\text { number }\end{array}$ & Cancer subsite & Treatment & Survival rate & $\begin{array}{l}\text { Organ } \\
\text { preservation rate }\end{array}$ \\
\hline \multirow[t]{2}{*}{$\overline{\text { VALCSG [11] }}$} & & 332 & Stage III/IV LAx & Surgery & $68 \%$ at $2 \mathrm{yr}$ & \\
\hline & & & & Induction $C / T+R T$ & $68 \%$ at $2 \mathrm{yr}$ & $64 \%$ at $2 \mathrm{yr}$ \\
\hline Malone et al. [25] & 1993-2000 & 40 & Stage III/IV BOT & OP+adj-CCRT & $74.7 \%$ at $2 \mathrm{yr}$ & - \\
\hline Sewnaik et al. [5] & 1985-1994 & 893 & $\mathrm{HPx}$ & Surgery and RT & $32 \%$ at $5 \mathrm{yr}$ & \\
\hline Adelstein et al. $[24,24]$ & 1989-2002 & 222 & All head and neck & CCRT & $65.7 \%$ at $5 \mathrm{yr}$ & $62.2 \%$ at $5 \mathrm{yr}$ \\
\hline \multirow[t]{2}{*}{ Soo et al. [4] } & & 119 & All head and neck & Surgery & $50 \%$ at $3 \mathrm{yr}^{\#}$ & \\
\hline & & & & CCRT & $40 \%$ at $3 \mathrm{yr}^{\#}$ & $45 \%$ at $3 \mathrm{yr}$ \\
\hline Hanna et al. [7] & 1996-2002 & 127 & OPx, LAx, HPx, OC & CCRT & $57 \%$ at $3 \mathrm{yr}$ & - \\
\hline Urba et al. [6] & & 59 & BOT, HPx & Induction $C / T+C C R T$ & $64 \%$ at $3 \mathrm{yr}$ & $52 \%$ at $3 \mathrm{yr}$ \\
\hline \multirow[t]{2}{*}{ Current series } & 1994-2004 & 395 & $\mathrm{HPx}$ & Surgery & $18.8 \%$ at $5 \mathrm{yr}$ & \\
\hline & & & & CCRT & $27 \%$ at $5 \mathrm{yr}$ & $\begin{array}{l}44.8 \% \text { at } 3 \mathrm{yr} \\
37 \% \text { at } 4 \mathrm{yr}\end{array}$ \\
\hline
\end{tabular}

\#: disease-free survival; Lax: larynx; BOT: base of tongue; HPx: hypopharynx; OPx: oropharynx; OC: oral cavity 
of betel quid chewing, smoking and/or alcohol drinking. Squamous cell carcinoma of upper aero-digestive tract (including oral cavity, pharynx, esophagus and lung) is the most common cancer that occurs in Taiwanese man, and the incidence of oral cavity cancer and esophageal cancer is increasing $13.1 \%$ and $4.1 \%$ respectively in ten years in Taiwan[32]. On the other hand, most of our patients have the habits of smoking, betel quid chewing and alcohol consumption, and the concept of field cancerization from Slaughter et al. [33] may explain the relative high incidence of second primary malignancy in our patients.

\section{Conclusion}

The majority of our hypopharyngeal cancer patients presented at stage IV. There was no survival difference between the organ preservation intended therapy and radical surgery groups. Patients who received CCRT had a better chance of survival with a preserved larynx compared with patients who received induction chemotherapy. Secondary cancer was a major cause of death. The median time to the development of a second primary malignancy was 2.64 years, with a $4.6 \%$ annual incidence. We suggest that organ preservation intended therapy, especially CCRT, should be considered first for patients with advanced hypopharyngeal cancer patients who refuse, or are unable to undergo, radical surgery.

\section{Acknowledgements \\ Grant Support: CMRPG360091}

\section{Author details \\ 'Department of Radiation Oncology, Hsinchu General Hospital, Hsin-Chu, Taiwan. ${ }^{2}$ Division of Hematology/Medical Oncology, Department of Internal Medicine, Chang Gung Memorial Hospital at Linkou, Taoyuan, Taiwan. ${ }^{3}$ Department of Otorhinolaryngology/Head and Neck Surgery, Chang Gung Memorial Hospital at Linkou, Taoyuan, Taiwan. ${ }^{4}$ Department of Radiation Oncology, Chang Gung Memorial Hospital at Linkou, Taoyuan, Taiwan. ${ }^{5}$ Taipei Chang Gung Head and Neck Oncology Group, Chang Gung Memorial Hospital at Linkou, Taoyuan, Taiwan. ${ }^{6}$ Department of Medicine, Chang Gung University, Taoyuan, Taiwan. ${ }^{7}$ Graduate Institute of Clinical Medical Science, Chang Gung University, Taoyuan, Taiwan.}

\section{Authors' contributions}

MFC and JTC designed and coordinated the study. Patient accrual and clinical data collection was done by all authors. Data analysis and treatment data collection was done by MFC and JTC. MFC prepared the manuscript. HW and JTC revised critically for important intellectual content. All authors read and approved the final manuscript.

\section{Competing interests}

The authors declare that they have no competing interests.

Received: 29 May 2010 Accepted: 7 October 2010

Published: 7 October 2010

\section{References}

1. Lajtman Z, Manestar D: A comparison of surgery and radiotherapy in the management of advanced pyriform fossa carcinoma. Clin Otolaryngol Allied Sci 2001, 26:59-61.
2. Lee NY, O'Meara W, Chan K, Della-Bianca C, Mechalakos JG, Zhung J, Wolden SL, Narayana A, Kraus D, Shah JP, Pfister DG: Concurrent chemotherapy and intensity-modulated radiotherapy for locoregionally advanced laryngeal and hypopharyngeal cancers. Int J Radiat Oncol Biol Phys 2007, 69:459-468.

3. Arriagada R, Eschwege F, Cachin Y, Richard JM: The value of combining radiotherapy with surgery in the treatment of hypopharyngeal and laryngeal cancers. Cancer 1983, 51:1819-1825.

4. Soo KC, Tan EH, Wee J, Lim D, Tai BC, Khoo ML, Goh C, Leong SS, Tan T, Fong KW, et al: Surgery and adjuvant radiotherapy vs concurrent chemoradiotherapy in stage III/IV nonmetastatic squamous cell head and neck cancer: a randomised comparison. Br J Cancer 2005, 93:279-286.

5. Sewnaik A, Hoorweg JJ, Knegt PP, Wieringa MH, van der Beek JM, Kerrebijn JD: Treatment of hypopharyngeal carcinoma: analysis of nationwide study in the Netherlands over a 10-year period. Clin Otolaryngol 2005, 30:52-57

6. Urba SG, Moon J, Giri PG, Adelstein DJ, Hanna E, Yoo GH, Leblanc M, Ensley JF, Schuller DE: Organ preservation for advanced resectable cancer of the base of tongue and hypopharynx: a Southwest Oncology Group Trial. J Clin Oncol 2005, 23:88-95.

7. Hanna E, Alexiou M, Morgan J, Badley J, Maddox AM, Penagaricano J, Fan CY, Breau R, Suen J: Intensive chemoradiotherapy as a primary treatment for organ preservation in patients with advanced cancer of the head and neck: efficacy, toxic effects, and limitations. Arch Otolaryngol Head Neck Surg 2004, 130:861-867.

8. Robbins KT, Fontanesi J, Wong FS, Vicario D, Seagren S, Kumar P, Weisman R, Pellitteri $P$, Thomas JR, Flick $P$, et al: A novel organ preservation protocol for advanced carcinoma of the larynx and pharynx. Arch Otolaryngol Head Neck Surg 1996, 122:853-857.

9. Rudat V, Pfreundner L, Hoppe F, Dietz A: Approaches to preserve larynx function in locally advanced laryngeal and hypopharyngeal cancer. Onkologie 2004, 27:368-375.

10. Zelefsky MJ, Kraus DH, Pfister DG, Raben A, Shah JP, Strong EW, Spiro RH, Bosl GJ, Harrison LB: Combined chemotherapy and radiotherapy versus surgery and postoperative radiotherapy for advanced hypopharyngeal cancer. Head Neck 1996, 18:405-411.

11. Induction chemotherapy plus radiation compared with surgery plus radiation in patients with advanced laryngeal cancer. The Department of Veterans Affairs Laryngeal Cancer Study Group. N Engl J Med 1991, 324:1685-1690.

12. Lefebvre JL, Chevalier D, Luboinski B, Kirkpatrick A, Collette L, Sahmoud T: Larynx preservation in pyriform sinus cancer: preliminary results of a European Organization for Research and Treatment of Cancer phase III trial. EORTC Head and Neck Cancer Cooperative Group. J Natl Cancer Inst 1996, 88:890-899.

13. Pignon JP, Bourhis J, Domenge C, Designe L: Chemotherapy added to locoregional treatment for head and neck squamous-cell carcinoma: three meta-analyses of updated individual data. MACH-NC Collaborative Group. Meta-Analysis of Chemotherapy on Head and Neck Cancer. Lancet 2000, 355:949-955.

14. Adelstein DJ, Sharan VM, Earle AS, Shah AC, Vlastou C, Haria CD, Damm C, Carter SG, Hines JD: Simultaneous versus sequential combined technique therapy for squamous cell head and neck cancer. Cancer 1990, 65:1685-1691.

15. Calais $G$, Alfonsi $M$, Bardet $E$, Sire $C$, Germain $T$, Bergerot $P$, Rhein $B$, Tortochaux J, Oudinot $P$, Bertrand $P$ : Randomized trial of radiation therapy versus concomitant chemotherapy and radiation therapy for advancedstage oropharynx carcinoma. J Natl Cancer Inst 1999, 91:2081-2086.

16. Wang HM, Wang CS, Chen JS, Chen IH, Liao CT, Chang TC: Cisplatin, tegafur, and leucovorin: a moderately effective and minimally toxic outpatient neoadjuvant chemotherapy for locally advanced squamous cell carcinoma of the head and neck. Cancer 2002, 94:2989-2995.

17. Cachin Y, Eschwege F: Combination of radiotherapy and surgery in the treatment of head and neck cancers. Cancer Treat Rev 1975, 2:177-191.

18. Featherstone CJ, Clarke S, Jackson MA, Shannon KF, McNeil EB, Tin MM, Clifford A, O'Brien CJ: Treatment of advanced cancer of the larynx and hypopharynx with chemoradiation. ANZ J Surg 2004, 74:554-558.

19. Garden AS, Harris J, Vokes EE, Forastiere AA, Ridge JA, Jones C, Horwitz EM, Glisson BS, Nabell L, Cooper JS, et al: Preliminary results of Radiation Therapy Oncology Group 97-03: a randomized phase ii trial of 
concurrent radiation and chemotherapy for advanced squamous cell carcinomas of the head and neck. J Clin Oncol 2004, 22:2856-2864.

20. Kim JG, Sohn SK, Kim DH, Baek JH, Jeon SB, Chae YS, Lee KB, Park JS, Sohn JH, Kim JC, Park IK: Phase II study of concurrent chemoradiotherapy with capecitabine and cisplatin in patients with locally advanced squamous cell carcinoma of the head and neck. Br J Cancer 2005, 93:1117-1121.

21. Lavertu P, Adelstein DJ, Saxton JP, Secic M, Eliachar I, Strome M, Larto MA, Wood BG: Aggressive concurrent chemoradiotherapy for squamous cell head and neck cancer: an 8-year single-institution experience. Arch Otolaryngol Head Neck Surg 1999, 125:142-148.

22. Razack MS, Sako K, Marchetta FC, Calamel P, Bakamjian V, Shedd DP: Carcinoma of the hypopharynx: success and failure. Am J Surg 1977, 134:489-491.

23. Rudat $\mathrm{V}$, Wannenmacher M: Role of multimodal treatment in oropharynx, larynx, and hypopharynx cancer. Semin Surg Oncol 2001, 20:66-74.

24. Adelstein DJ, Saxton JP, Rybicki LA, Esclamado RM, Wood BG, Strome M, Lavertu P, Lorenz RR, Carroll MA: Multiagent concurrent chemoradiotherapy for locoregionally advanced squamous cell head and neck cancer: mature results from a single institution. J Clin Oncol 2006, 24:1064-1071.

25. Malone JP, Stephens JA, Grecula JC, Rhoades CA, Ghaheri BA, Schuller DE: Disease control, survival, and functional outcome after multimodal treatment for advanced-stage tongue base cancer. Head Neck 2004, 26:561-572.

26. Ghi MG, Paccagnella A, D'Amanzo P, Mione CA, Fasan S, Paro S, Mastromauro C, Carnuccio R, Turcato G, Gatti C, et al: Neoadjuvant docetaxel, cisplatin, 5-fluorouracil before concurrent chemoradiotherapy in locally advanced squamous cell carcinoma of the head and neck versus concomitant chemoradiotherapy: a phase II feasibility study. Int J Radiat Oncol Biol Phys 2004, 59:481-487.

27. Forastiere AA, Goepfert H, Maor M, Pajak TF, Weber R, Morrison W, Glisson B, Trotti A, Ridge JA, Chao C, et al: Concurrent chemotherapy and radiotherapy for organ preservation in advanced laryngeal cancer. $N$ Engl J Med 2003, 349:2091-2098.

28. Studer G, Lutolf UM, Davis JB, Glanzmann C: IMRT in hypopharyngeal tumors. Strahlenther Onkol 2006, 182:331-335.

29. Studer G, Peponi E, Kloeck S, Dossenbach T, Huber G, Glanzmann C Surviving hypopharynx-larynx carcinoma in the era of IMRT. Int I Radiat Oncol Biol Phys 77:1391-1396.

30. Eisbruch A, Schwartz M, Rasch C, Vineberg K, Damen E, Van As CJ, Marsh R, Pameijer FA, Balm AJ: Dysphagia and aspiration after chemoradiotherapy for head-and-neck cancer: which anatomic structures are affected and can they be spared by IMRT? Int I Radiat Oncol Biol Phys 2004, 60:1425-1439.

31. Carrara-de Angelis E, Feher O, Barros AP, Nishimoto IN, Kowalski LP: Voice and swallowing in patients enrolled in a larynx preservation trial. Arch Otolaryngol Head Neck Surg 2003, 129:733-738.

32. Department of Health: the Executive Yuan, February 2010. Cancer registry: annual report in Taiwan area in 2007.

33. Slaughter DP, Southwick HW, Smejkal W: Field cancerization in oral stratified squamous epithelium; clinical implications of multicentric origin. Cancer 1953, 6:963-968.

doi:10.1186/1748-717X-5-91

Cite this article as: Chang et al:: Treatment results for hypopharyngeal cancer by different treatment strategies and its secondary primary- an experience in Taiwan. Radiation Oncology 2010 5:91.

\section{Submit your next manuscript to BioMed Central and take full advantage of:}

- Convenient online submission

- Thorough peer review

- No space constraints or color figure charges

- Immediate publication on acceptance

- Inclusion in PubMed, CAS, Scopus and Google Scholar

- Research which is freely available for redistribution

Submit your manuscript at www.biomedcentral.com/submit
C Biomed Central 